\title{
Análisis de riesgos higiénicos en aserraderos (Parte I)
}

\author{
Hygienic Risk Analysis in Sawmills (Part I)
}

\author{
Lourdes Medina-Escobar' \\ Gabriela Hernández-Gómez² \\ Gabriela Rodríguez-Zamora ${ }^{3}$ \\ Carlos Mata-Montero ${ }^{4}$
}

Fecha de recepción: 26 de febrero del 2014

Fecha de aprobación: 25 de mayo del 2014

Medina-Escobar, L; Hernández-Gómez, G;

Rodríguez-Zamora, G; Mata-Montero, C.

Análisis de riesgos higiénicos en aserraderos

(Parte I). Tecnología en Marcha. Vol. 27, № 4,

Octubre-Diciembre. Pág 30-40.

I Máster en Química Industrial, Escuela de Ingeniería en Seguridad Laboral e Higiene Ambiental, Instituto Tecnológico de Costa Rica. Cartago, Costa Rica. Tel. (506) 2550-9363. Correo electrónico: mmedina@itcr.ac.cr

2 Ingeniera en Seguridad Laboral e Higiene Ambiental, Laboratorio de Higiene Analítica, Instituto Tecnológico de Costa Rica. Cartago, Costa Rica. Tel. (506) 2550-2800. Correo electrónico: mgabrielahdez@gmail.com

3 Bachiller en Química, Escuela de Ingeniería en Seguridad Laboral e Higiene Ambiental, Instituto Tecnológico de Costa Rica. Cartago, Costa Rica. Tel. (506) 2550-9363. Correo electrónico: garodriguez@itcr.ac.cr

4 Ingeniero Industrial, MAP. Instituto Tecnológico de Costa Rica. Cartago, Costa Rica. Tel. (506) 2550-27II. Correo electrónico: camata@itcrac.cr 


\section{Palabras clave}

Aserraderos; exposición ocupacional; polvo de madera; agentes biológicos.

\section{Resumen}

Las enfermedades respiratorias y el cáncer nasal se encuentran entre las afecciones ocupacionales asociadas con la exposición polvo de madera. El presente estudio permitió valorar de forma exploratoria algunos agentes químicos y biológicos que podrían estar afectando la salud de los trabajadores de los aserraderos. Para las evaluaciones de la exposición a polvo de madera se utilizó equipo de medición para la recolección de muestras según el método MDHS I4/3 (2000), que fueron analizadas posteriormente en el Laboratorio de Higiene Analítica; las muestras de agentes biológicos se analizaron según Pouch (2003) en el Laboratorio de Alimentos de la Universidad de Costa Rica.

Las concentraciones de polvo de madera se encontraron en un rango de 0,09-28,9 mg/ $\mathrm{m}^{3}$, con un MLE estimado de $2,33 \mathrm{mg} / \mathrm{m}^{3}$, superando la normativa nacional e internacional. Se encontraron especies de hongos y bacterias en las muestras de polvo de madera: Aspergillus, Penicillium y Bacillum. La caracterización más detallada de la presencia de agentes biológicos en el polvo de madera es un área de investigación novedosa en el país, que debe ser retomada en investigaciones futuras para una mejor comprensión del papel que desempeñan en las patologías respiratorias e inmunológicas del personal expuesto.

\section{Key words}

Sawmills; occupational exposure; wood dust; biological agents.

\section{Abstract}

Respiratory and nasal cancers are among the diseases associated with occupational exposure to wood dust. This study allowed assessing, in an exploratory way, some chemical and biological agents that could be affecting the health of the mill workers. For the wood dust exposure assessments, measurement equipment to collect samples according to the method MDHS I4/3 (2000) was used, subsequently analyzed in the Laboratory of Analytical Hygiene of the ITCR and biological agents samples were analyzed according to Pouch (2003) in the Food Laboratory of the University of Costa Rica.

The wood dust concentrations were found in a range of 0.09 to $28.9 \mathrm{mg} / \mathrm{m} 3$, with an MLE estimate of $2.33 \mathrm{mg} / \mathrm{m} 3$ exceeding national and international regulations. Species of fungi and bacteria were found in samples of wood dust: Aspergillus, Bacillum, and Penicillium.

The detailed characterization of the presence of biological agents in the wood dust is a novel research area in the country to be taken up in future research for better understanding of their role in respiratory and immune pathology of exposed workers.

\section{Introducción}

La exposición a polvo de madera incluye múltiples operaciones, que van desde la corta de los árboles hasta el descortezado, el aserrío, el corte, el taladrado, el lijado, el acabado y la manufactura de productos de madera. Esta exposición se caracteriza por las dimensiones de la intensidad (usualmente expresada en miligramos de polvo de madera por metro cúbico de aire), patrón de tiempo de exposición y composición química, y características físicas del polvo de madera.
Como ocurre con muchas máquinas para trabajar la madera, la cantidad de polvo generado por las operaciones de aserrado mecánico está influenciada por la velocidad de la acción de aserrío, el ángulo de corte con respecto a la veta de la madera y la nitidez y el ancho de la hoja. En consecuencia, las cuchillas afiladas y delgadas producen menos polvo de madera por volumen porque el tamaño de la ranura de corte hecho en la madera es más estrecho, sin embargo, es probable que los tamaños de las partículas también sean menores (IARC, 1995). 
La Agencia Internacional para la Investigación sobre el Cáncer ha clasificado el polvo de madera como cancerígeno para los seres humanos (Grupo I) (IARC, 1995). El cáncer nasal ha sido vinculado como un peligro significativo del trabajo con madera, particularmente en la elaboración de muebles y en labores con maderas duras (Kauppinen, Vincent, Liukkpnen, Grzebyk, Kauppinen y Welling 2006; Spee, van de Rijdt-van Hoof, van Hoof, Noy y Kromhout, 2007).

La toxicidad de la madera y sus efectos irritantes sobre la piel y el aparato respiratorio en humanos han sido ampliamente documentados (Hausen y Rothernborg, 1981; Woods y Calnan, 1976). El Comité Científico de la Comisión Europea sobre límites de exposición profesional ha informado que los trabajadores expuestos a polvo de madera dura o blanda en concentraciones superiores a 0,5 mg/ $\mathrm{m}^{3}$ han mostrado deterioro significativo de la salud (Nigel, Dilworth y Summers, 2007). En general, la exposición a polvo de madera deteriora la función pulmonar, aumenta la prevalencia de enfermedades respiratorias y agrava las ya existentes (Osman y Pala, 2009).

Lamentablemente, la investigación de estos efectos ha estado enfocada en las especies de madera utilizadas en países desarrollados con climas templados, y se han generado pocos estudios en relación con especies tropicales, de las cuales, sin embargo, se sospechan mayores efectos irritantes. Para el caso costarricense, la información disponible en cuanto a la caracterización de las especies autóctonas se ha limitado a la valoración de las condiciones mecánicas y estructurales más que a sus componentes químicos y efectos biológicos.

Con respecto a este último aspecto, se encontraron estudios (Oppliger, Rusca, Charrie're, Vu Duc y Droz, 2005) en los que se reportan concentraciones altas de hongos (35 0000UFC/m ${ }^{3}$ ) y bacterias (Gram negativas y endotoxinas) en aserraderos, incluyendo predominantemente Penicillium sp. y la familia de las Pseudomonadacea (bacterias Gram-positiva y Gram-negativa).

En el presente informe se documentan los resultados de una investigación exploratoria, la cual ofrece una comprensión inicial de la situación actual en Costa Rica en relación con la exposición ocupacional a riesgos higiénicos, en una muestra de empresas dedicadas al aserrío de maderas nacionales.

\section{Materiales y métodos}

Se realizó una investigación con elementos descriptivos, ya que permitió especificar las causas de mayor relevancia en la ocurrencia de enfermedades en la actividad de aserrío de madera.

La selección de la muestra fue no probabilística, considerando como marco poblacional la lista de empresas aseguradas por el Instituto Nacional de Seguros (INS) bajo el seguro de Riesgos del Trabajo para la actividad de aserrado y acepilladura de madera. Se tomaron como muestra de estudio 14 aserraderos que actualmente tienen trabajadores asegurados y se consideró cada empresa como una de las unidades de análisis sobre las cuales se realizó la valoración de los factores de riesgo. Las empresas estaban distribuidas por todo el país.

A partir de encuestas higiénicas se recopiló información sobre los aserraderos, número de trabajadores, jornada laboral, descripción del proceso, tareas que se realizan y puestos de trabajo más críticos (de mayor exposición), entre otros aspectos.

\section{Exposición a polvo de madera}

Se utilizó como referencia el método MDHS 14/3: General methods for sampling and gravimetric analysis of respirable and inhalable dust, para la recolección de las muestras y la cuantificación de los niveles de exposición ocupacional a polvo de madera (Health and Safety Executive, 2000). Para efectuar los muestreos se emplearon filtros de PVC de 25 $\mathrm{mm}$ de diámetro, con soporte de celulosa, cabezas IOM, bombas de muestreo personal de alto caudal, tubos flexibles, etc.

Dada la variabilidad de los procesos y los objetivos del estudio, se adoptaron estrategias de peor caso para definir actividades críticas y trabajadores, que a juicio de los investigadores estuvieran expuestos a polvo de madera o presentaran riesgo de compromiso ergonómico durante la realización de las tareas.

Las muestras de exposición ocupacional a polvo de madera fueron analizadas en el Laboratorio de Higiene Analítica, ubicado en la Escuela de Ingeniería en Seguridad Laboral e Higiene Ambiental del Instituto Tecnológico de Costa Rica.

Se realizaron análisis estadísticos con el fin de caracterizar la exposición a polvo de madera, 
particularmente su fracción inhalable por tareas y empresas. Esta caracterización contempla información sobre medidas de tendencia central, medidas de dispersión, comparación de las tareas en diferentes escenarios ocupacionales y otros análisis que, según la revisión de la literatura y el comportamiento de los datos, permitieron identificar factores que pudieron influir en la exposición. Entre las herramientas estadísticas utilizadas están los histogramas de frecuencia y cálculos de parámetros de tendencia central según tipo de distribución teórica de los datos, entre otras.

La recolección de muestras de polvo de madera para el análisis biológico de conteo de poblaciones se llevó a cabo al final del muestreo y se tomó de los residuos de madera depositados alrededor de la sierra principal. La identificación de los microorganismos se realizó bajo el método Pouch 2003: Compendium of methods for the examination of food. APHA. La cuantificación de los hongos y bacterias se llevó a cabo en el Laboratorio Alimentos de la Universidad de Costa Rica.

\section{Resultados y discusión}

se contó con la participación de 14 aserraderos, que se visitaron en una sola ocasión. Las evaluaciones se realizaron en localidades ubicadas dentro y fuera de la Gran Área Metropolitana. Para mantener la confidencialidad de los aserraderos participantes, los datos se codificaron con una letra (C) y números según la cantidad de empresas ( I- |4).

\section{Exposición a polvo de madera}

Se analizaron un total de 38 muestras correspondientes a 28 trabajadores en 13 empresas participantes (ver cuadro I). Durante el período de evaluación, las tareas de aserrío se centraron en la operación de sierras principales, el manejo de máquinas para procesar piezas de madera (como canteadoras, cepilladoras, machimbradoras, motosierras, etc.) y la recepción de madera luego de ser procesada.

Entre las maderas que se registró que se procesaron durante el muestreo de los aserraderos se encontró la presencia principalmente de laurel y melina. También algunos encargados mencionaron usar botarrama, cativo, lagartillo, pilón, gallinazo, reseco y terminalia.
Cuadro I. Concentraciones de polvo de madera.

\begin{tabular}{|c|c|c|}
\hline $\begin{array}{l}\text { Código de } \\
\text { muestra }\end{array}$ & $\begin{array}{l}\text { Código de } \\
\text { empresa }\end{array}$ & $\begin{array}{c}\text { Concentración } \\
\mathrm{mg} / \mathrm{m}^{3}\end{array}$ \\
\hline 149 & $\mathrm{Cl}$ & 1,82 \\
\hline 145 & $\mathrm{C} 2$ & 0,77 \\
\hline 151 & $C 2$ & ND \\
\hline 154 & $\mathrm{C2}$ & 7,56 \\
\hline 155 & $C 2$ & 5,97 \\
\hline 169 & $\mathrm{C} 3$ & 1,70 \\
\hline 170 & $\mathrm{C} 3$ & 1,35 \\
\hline 161 & C4 & 0,42 \\
\hline 162 & C4 & 4,77 \\
\hline 165 & $\mathrm{C} 5$ & 0,09 \\
\hline 166 & $\mathrm{C} 5$ & 28,9 \\
\hline 167 & C5 & 0,55 \\
\hline 168 & $\mathrm{C} 5$ & 0,86 \\
\hline 179 & $\mathrm{C5}$ & ND \\
\hline 163 & C6 & 1,09 \\
\hline 164 & C6 & 1,09 \\
\hline 171 & C6 & 0,77 \\
\hline 172 & $\mathrm{C} 6$ & 4,79 \\
\hline 174 & C6 & 11,69 \\
\hline 182 & C7 & 4,26 \\
\hline 183 & C7 & ND \\
\hline 184 & C7 & 0,68 \\
\hline 201 & C8 & 2,64 \\
\hline 202 & C8 & 1,28 \\
\hline 208 & $\mathrm{ClO}$ & 0,94 \\
\hline 209 & $\mathrm{ClO}$ & 1,71 \\
\hline 210 & $\mathrm{ClO}$ & 0,69 \\
\hline 211 & $\mathrm{ClO}$ & 0,54 \\
\hline 223 & $\mathrm{Cll}$ & 2,78 \\
\hline 218 & $\mathrm{Cll}$ & 0,47 \\
\hline 219 & $\mathrm{Cll}$ & 2,86 \\
\hline 227 & $\mathrm{Cl} 2$ & 0,93 \\
\hline 228 & $\mathrm{Cl} 2$ & 0,77 \\
\hline 229 & $\mathrm{Cl} 2$ & 0,64 \\
\hline 263 & $\mathrm{Cl} 3$ & ND \\
\hline 264 & $\mathrm{Cl} 3$ & 16,7 \\
\hline 258 & $\mathrm{Cl} 4$ & 1,73 \\
\hline 259 & $\mathrm{Cl} 4$ & 0,67 \\
\hline
\end{tabular}


Los resultados se compararon con los valores umbrales límite de exposición ponderados en el tiempo (TLV-TWA) propuestos por la asociación Americana de Higienistas Industriales Gubernamentales (ACGIH, 20II). Debido a que se reportan jornadas laborales diferentes entre ellas, se aplicó la relación sugerida por Brief y Scala para la reducción del valor de polvo de madera, a fin de poder hacer inferencias sobre el cumplimiento (Brief y Scala, 1975).

Para efecto de realizar los cálculos necesarios para hacer inferencias estadísticas, en aquellos casos donde se reportaron concentraciones por debajo del límite de detección establecido, se tomó como base el trabajo de Hornung y Reed, en el cual se estudia el error asociado con la estimación de la media de exposición cuando se tienen lecturas inferiores a los límites de detección y se introdujo como valor de la concentración para estas lecturas el equivalente a 0,5 veces el límite de detección (Hornung y Reed, 1990).

La figura I muestra la distribución sin tratamiento estadístico de los resultados. Es posible observar con claridad un importante sesgo a la izquierda, patrón que coincide con las distribuciones referidas en la literatura para los valores de concentraciones en ambientes laborales (Gilbert, 1987).

El comportamiento de los datos fue mejor descrito por una distribución log normal, congruente con

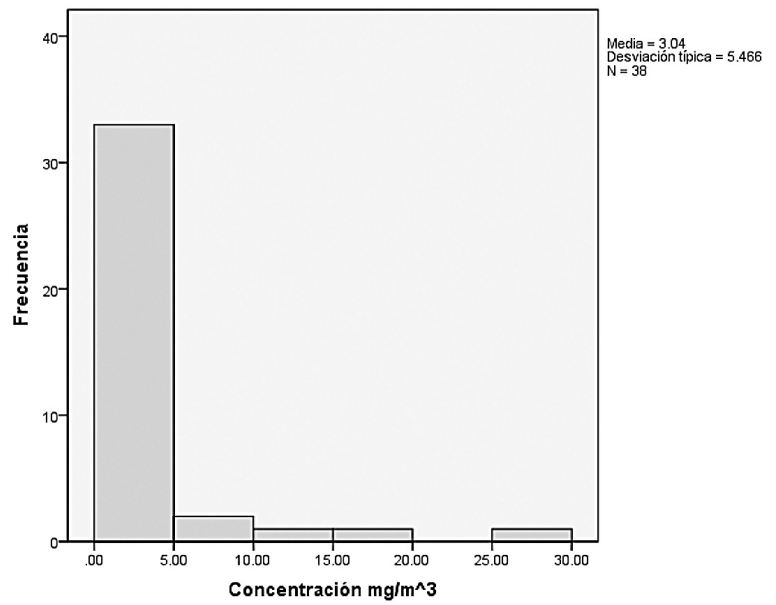

Figura I. Distribución de las concentraciones de polvo de madera en $\mathrm{mg} / \mathrm{m}^{3}$ los resultados de las investigaciones de Rappaport y Selvin para este tipo de datos (ver figura 2) (Rappaport y Selvin, 1987). Por lo anterior, el análisis de cumplimiento con la norma se basó en parámetros que pueden ser convenientemente comparados con este tipo de distribución.

La figura 3 ofrece los datos individuales de concentración obtenidos para las 38 muestras. Se puede observar que la mayor dispersión de los datos se presentó en la empresa C5, la cual además presentó la concentración individual más alta del conjunto de datos $\left(28,9 \mathrm{mg} / \mathrm{m}^{3}\right)$, superando el valor máximo permitido para polvo de madera, que en el caso particular de esta empresa es de 0,70 $\mathrm{mg} / \mathrm{m}^{3}$. El trabajador que reportó este valor tenía el puesto de ayudante de aserrío; sus actividades consistieron en recibir piezas de madera recién cortadas y trasladarlas al área de elaboración de tarimas, también se introdujo en la fosa ubicada debajo de la sierra vertical para remover el aserrín con pala. Durante todas las actividades que efectuó no usó equipo de protección respiratoria.

En el cuadro 2 se registra el número de trabajadores y muestras de polvo de madera en la fracción inhalable, así como las medidas de tendencia central de cada empresa considerada en el estudio. Se calcularon los límites de confiabilidad al 95\% en torno al MLE (estimador de máxima probabilidad para una distribución log normal), con el fin de contar con una estimación probabilística.

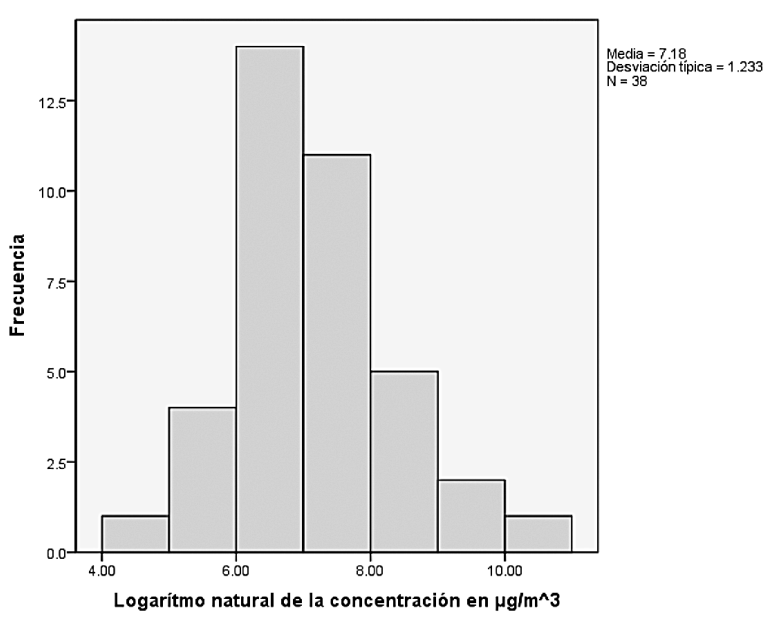

Figura 2. Distribución de las concentraciones de polvo de madera en $\mathrm{mg} / \mathrm{m} 3$ transformadas a logaritmo natural. 


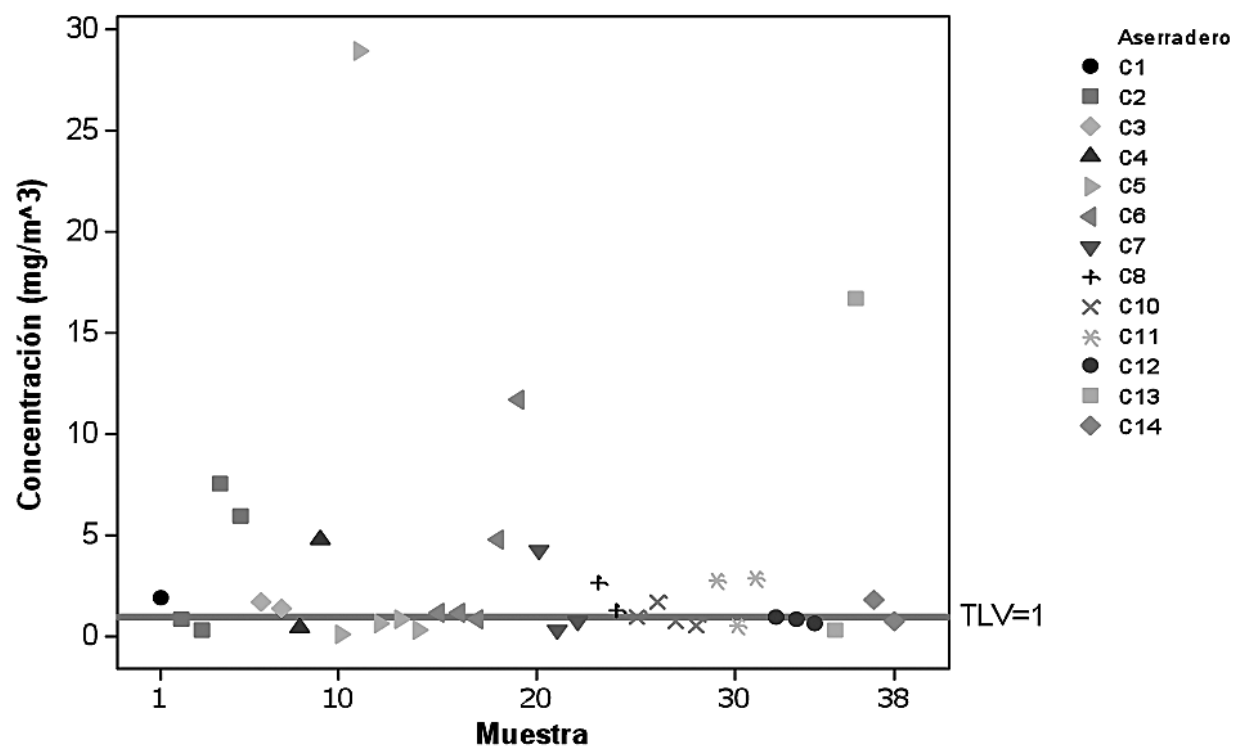

Figura 3. Dispersión de datos de las concentraciones de polvo de madera por aserradero.

Cuadro 2. Medidas de tendencia central de exposición ocupacional a polvo de madera fracción inhalable, por empresa.

\begin{tabular}{|c|c|c|c|c|c|c|c|}
\hline Empresa & $N^{\circ}$ muestras & $\begin{array}{c}\mathrm{N}^{\circ} \\
\text { trabajadores }\end{array}$ & $M^{a}(D G E)^{b}$ & $\mathrm{LIC}^{\mathrm{c}}$ & $M^{\prime} E^{d}$ & $\mathrm{LSC}^{\mathrm{e}}$ & $\mathrm{LEO}^{f}$ \\
\hline $\mathrm{C} 2$ & 4 & 3 & I,03 (4,80) & 0,82 & 053 & $>1000$ & 0,63 \\
\hline C3 & 2 & I & I,52 (1,18) & 1,08 & 1,54 & 2,43 & 0,70 \\
\hline C4 & 2 & । & ।,35 (5,55) & 0,41 & 5,88 & $>1000$ & 0,70 \\
\hline C5 & 5 & 4 & $0,52(9,01)$ & 0,73 & 5,83 & $>1000$ & 0,70 \\
\hline C6 & 5 & 4 & I,53 (3,22) & $1,2 \mid$ & 3,04 & 73,6 & 0,59 \\
\hline$C 7$ & 3 & 2 & $0,98(3,76)$ & 0,59 & 2,36 & $>1000$ & 1,00 \\
\hline $\mathrm{C} 8$ & 2 & 2 & I,84 (I,67) & 0,90 & 2,10 & 59,2 & 0,70 \\
\hline $\mathrm{ClO}$ & 4 & 2 & $0,85(1,65)$ & 0,60 & 0,96 & 3,76 & 0,70 \\
\hline $\mathrm{ClI}$ & 3 & 2 & I, $10(2,83)$ & 0,63 & 1,89 & $>1000$ & 0,63 \\
\hline $\mathrm{Cl} 2$ & 3 & 2 & $0,79(1,20)$ & 0,62 & 0,80 & 1,23 & 0,59 \\
\hline $\mathrm{Cl} 3$ & 2 & 2 & $2,24(17,0)$ & 0,93 & 124 & $>1000$ & 0,76 \\
\hline $\mathrm{Cl} 4$ & 2 & 2 & ।,08 (1,95) & 0,48 & 1,35 & 585 & 0,83 \\
\hline General & 38 & 28 & I,09 (3,43) & 1,58 & 2,33 & 3,99 & $-{ }^{*}$ \\
\hline \multicolumn{8}{|c|}{ 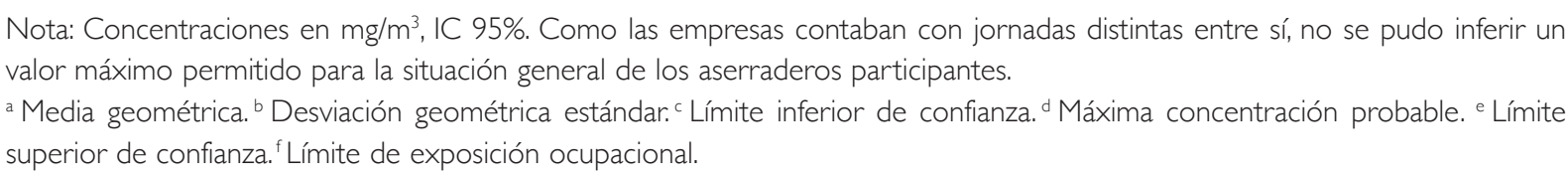 } \\
\hline
\end{tabular}


Puede observarse que para las empresas C2, C3, C5, C6, C8, ClI, Cl2 y Cl3 los intervalos de confianza para el MLE se encontraron por encima del TLV-TWA corregido en cada uno de los casos (dado que las jornadas variaban entre las empresas), lo cual indica una situación de sobreexposición.

El aserradero $\mathrm{Cl}$ tuvo solamente una muestra con una concentración de $1,82 \mathrm{mg} / \mathrm{m}^{3}$, la cual superó el límite de exposición ocupacional calculado para esta empresa $\left(0,83 \mathrm{mg} / \mathrm{m}^{3}\right)$. En el caso de los aserraderos C4, C7, ClO y $\mathrm{Cl} 4$, se encontraron valores que no permiten tomar una decisión (con un 95\% de confianza) sobre la situación de cumplimiento con la norma, por lo que no se descarta la posibilidad de condiciones de sobreexposición.
No se reportó ningún dato de exposición ocupacional a polvo de madera en la empresa C9. El día de la visita, la sierra principal estaba en reparación y no se estaban llevando a cabo otras labores que generaran exposición a este agente químico.

La figura 4 evidencia algunas tareas tomadas en cuenta en los muestreos de polvo de madera.

Se agruparon las concentraciones por tarea, con el propósito de caracterizar mejor la exposición ocupacional a polvo de madera en la fracción inhalable e identificar aquellas actividades realizadas por los trabajadores que podrían ser un riesgo, tal como se presenta en el cuadro 3.

El número de muestras y de trabajadores evaluados, así como las medidas de tendencia central estimadas para las tres tareas, se registran en el cuadro 4.

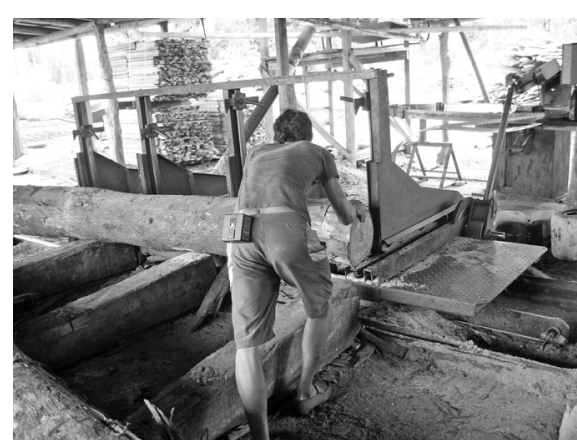

(a)

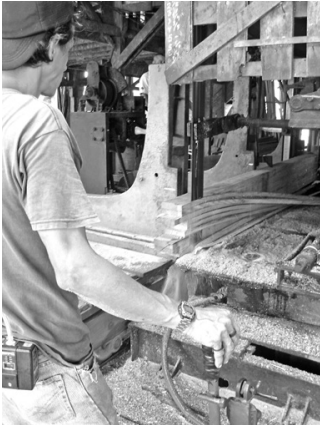

(b)

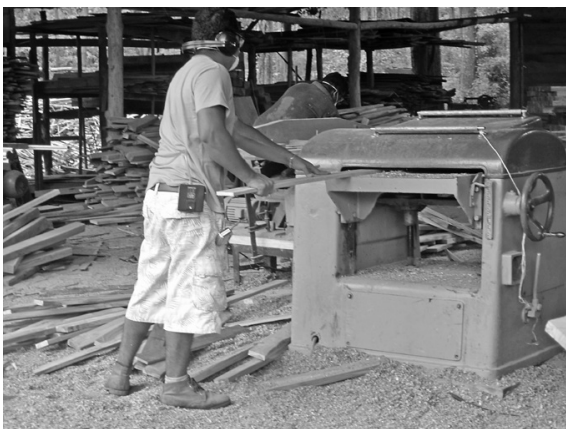

(c)

Figura 4. Tareas de aserrío con exposición ocupacional a polvo de madera.

Cuadro 3. Descripción de las tareas evaluadas para polvo de madera.

\begin{tabular}{|c|c|c|c|c|}
\hline Tarea & Descripción & EPP & No. observaciones & $\begin{array}{l}N^{0} \text { trabajadores } \\
\text { por aserraderos }\end{array}$ \\
\hline $\begin{array}{l}\text { Equipo } \\
\text { eléctrico }\end{array}$ & $\begin{array}{l}\text { Uso de machimbradora, metabo con punta de lija, } \\
\text { sierra de mesa, canteadora, multifuncional, sierra } \\
\text { múltiple, motosierra, cepilladora. }\end{array}$ & $\begin{array}{l}\text { Tres con mascarilla, } \\
\text { cinco sin equipo de } \\
\text { protección personal } \\
\text { (EPP) respiratoria }\end{array}$ & 8 & $\begin{array}{c}\text { C2:2, C3:1, C5:2, } \\
\text { C6:2, C1 I:I }\end{array}$ \\
\hline $\begin{array}{l}\text { Operador } \\
\text { sierra }\end{array}$ & $\begin{array}{l}\text { Aserrío de madera, limpieza de sierra (manual o } \\
\text { con aire comprimido), recoge aserrín, cambio de } \\
\text { cinta de la sierra, apila reglas. }\end{array}$ & $\begin{array}{l}\text { Uno con mascarilla, } \\
\text { nueve sin EPP } \\
\text { respiratorio }\end{array}$ & 10 & $\begin{array}{l}\text { C2, C4, C5, C6, } \\
\text { C7, C I0, CI2, CI3, } \\
\text { CI4 (un trabajador } \\
\text { por empresa) }\end{array}$ \\
\hline $\begin{array}{c}\text { Recepción } \\
\text { de piezas }\end{array}$ & $\begin{array}{l}\text { Recibo de piezas procesadas (semibloqueadora, } \\
\text { sierra, canteadora), ayudante de aserradero, sube } \\
\text { tronco al carro, hace limpieza de los alrededores } \\
\text { de la sierra (escoba y rastrillo), limpieza de tucas } \\
\text { con machete y cepillo de raíz. }\end{array}$ & $\begin{array}{l}\text { Diez sin EPP } \\
\text { respiratorio }\end{array}$ & 10 & $\begin{array}{l}\text { CI:I, C5:I, C6:I, } \\
\text { C7:I, C8:2, C10:1, } \\
\text { CI2:I, CI3:I, } \\
\text { CI4:I }\end{array}$ \\
\hline
\end{tabular}


Cuadro 4. Medidas de tendencia central de exposición ocupacional a polvo de madera fracción inhalable, por tarea.

\begin{tabular}{|c|c|c|c|c|c|c|}
\hline Tarea & $N^{\circ}$ muestras & $N^{0}$ trabajadores & MG $^{\mathrm{a}}(\mathrm{DGE})^{\mathrm{b}}$ & LIC $^{\mathrm{c}}$ & MLE $^{\mathrm{d}}$ & LSC $^{\mathrm{e}}$ \\
\hline Equipo eléctrico & 12 & 8 & $1,41(3,03)$ & 1,46 & 2,60 & 7,47 \\
\hline Operador sierra & 13 & 10 & $0,69(2,88)$ & 0,73 & 1,21 & 2,95 \\
\hline Recepción de piezas & 13 & 11 & $1,41(4,04)$ & 1,77 & 3,74 & 17,9 \\
\hline
\end{tabular}

Nota: Concentraciones en $\mathrm{mg} / \mathrm{m}^{3}$, IC 95\%. Como las empresas contaban con jornadas distintas entre sí, no se pudo inferir un valor máximo permitido para cada tarea.

a Media geométrica. ${ }^{b}$ Desviación geométrica estándar. ${ }^{c}$ Límite inferior de confianza. ${ }^{d}$ Máxima concentración probable. ${ }^{\text {e Límite }}$ superior de confianza.

Como se ha mencionado anteriormente, los aserraderos que participaron en el estudio tuvieron jornadas de trabajo variadas, lo que condujo a que los datos estimados por tarea no pudieran compararse directamente contra un único valor límite de exposición ocupacional (LEO), por lo que se tomó como criterio de comparación el TLV-TWA sin corregir de este agente químico.

Según los datos mostrados en la tabla anterior, los intervalos de confianza para el MLE de las tareas relacionadas con el uso de equipo eléctrico y el recibo de piezas procesadas se encontraron por encima del TLV-TWA sin corregir (igual a I mg/ $\mathrm{m}^{3}$ ). Con respecto a los 10 operadores de sierra principal, no se pudo tomar una decisión sobre la situación de cumplimiento con la norma, por lo que no se descarta la posibilidad de que existan condiciones de sobreexposición.

En el tema del control de polvo de madera, algunas empresas cuentan con sistemas de bandas transportadoras para el traslado de polvo y aserrín que se genera en el proceso de aserrío. Sin embargo, la estructura del silo donde se almacena el desecho está fabricada con tablones de madera o láminas metálicas con rendijas y espacios que permiten el escape de polvo de madera hacia el exterior (figura 5a). Dependiendo de la dirección del viento y la ubicación de los puestos de trabajo, esto podría afectar al trabajador.

Además, se registraron otros casos de aserraderos que depositan en la parte posterior de la planta todo el aserrín que se genera durante la actividad de aserrío, tal y como se evidencia en la figura 5(b) y $5(c)$.
Finalmente, debe considerarse que el método de análisis empleado en este estudio para caracterizar la exposición ocupacional a polvo de madera no permitió discriminar los componentes del polvo capturado en el filtro, por lo que se considera poco probable que las muestras solo tuvieran polvo de madera, lo cual ha sido discutido por varios autores (Teschke, Demers, Davies, Kennedy, Marion y Leung, 1999; Demers, Teschke, Davies, Kennedy y Leung, 2000; Fransman, McLean, Douwes, Demers, Leung y Pearce, 2003).

La infraestructura de los locales de los aserraderos constaba de techo metálico y pocas divisiones de tablones de madera o láminas de metal, las estructuras no contaban con paredes que detuvieran el ingreso de polvo desde el exterior. Por lo tanto, los trabajadores podrían exponerse fácilmente a polvo proveniente del ambiente.

\section{Agentes biológicos}

Tanto el polvo de madera como los microorganismos (hongos, bacterias, mohos, etc.) han sido sugeridos como causantes de asma ocupacional y otros síntomas respiratorios. Se ha realizado una cantidad importante de estudios con el fin de recopilar información sobre los tipos de microorganismos presentes en las diversas especies de madera, así como las concentraciones y efectos asociados con la salud de los trabajadores de aserraderos.

Con el fin describir de forma exploratoria la situación en Costa Rica, se analizó un total de siete muestras de polvo de madera tomadas de la salida de la sierra en diferentes aserraderos para identificar 


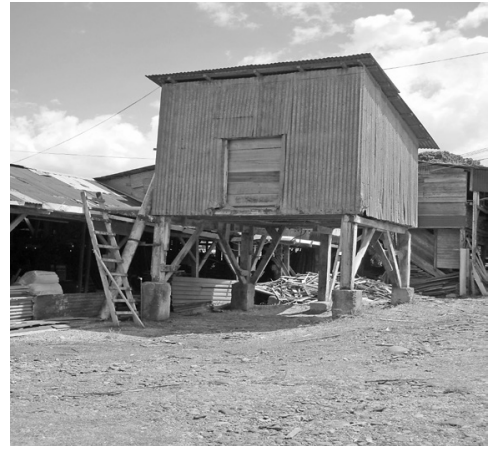

(a)

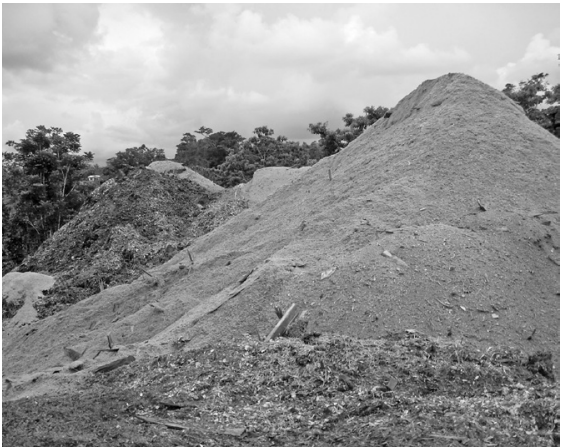

(b)

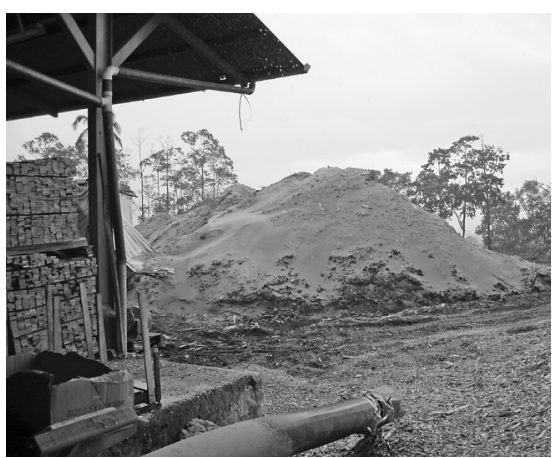

(c)

Figura 5. Almacenamiento de aserrín y mermas del proceso de aserrío.

posibles patógenos que pudieran estar agravando la exposición de los trabajadores.

En el cuadro 5 se pueden observar los diversos tipos de hongos y bacterias encontradas en las diferentes muestras de polvo de madera recolectadas.

El Aspergillus es un miembro del phylum Ascomycota. Hay más de 185 especies conocidas, de las cuales 20 se sabe que son perjudiciales para los seres humanos y otros animales. El subtipo más común asociado a infecciones de los senos paranasales y con la Aspergilosis es Aspergillus fumigatus. Los síntomas incluyen fiebre, tos, dolor de pecho o falta de aire. Por lo general, solo los pacientes que ya tienen sistemas inmunitarios debilitados o que sufren otras enfermedades pulmonares son susceptibles (Moro y Okiki, 2003; Land, Sostari, Fuchs, Lundstrom y Hult, 1989).

Las especies de Penicillium son hongos ubicuos que se encuentran en el suelo como saprófitos en vegetación podrida, causando la descomposición de materia vegetal como granos y productos alimentarios. Estos han sido previamente asociados tanto con asma como con alveolitis alérgica extrínseca (Halpin, 1994).

Los Bacillus con un género de bacterias con una gran diversidad. Pueden causar una gran cantidad de infecciones, desde las del oído hasta la meningitis y del tracto urinario hasta la septicemia. En la mayoría de los casos, se producen como infecciones secundarias en huéspedes inmuno-comprometidos o previamente afectados. Ellos pueden exacerbar la infección anterior mediante la producción de toxinas que dañan los tejidos o metabolitos que interfieren con el tratamiento. La enfermedad más conocida provocada por el bacilo es el ántrax, que es causado por la especie Bacillus anthracis (Enciclopedia Británica; Hameed, Khoder y Farag, 2000).

Solo para los aserraderos C-I y C-2 se realizó la cuantificación de la cantidad de unidades formadoras de colonias por gramo de madera, encontrándose valores de $1,3 \times 10^{3}$ y $2,8 \times 10^{5}$ respectivamente. Todas las especies de bacterias y hongos han sido reportadas en estudios similares (Oppigler et al., 2005; Douwes, Thorne, Pearce y Heederik, 2003).

Cuadro 5. Identificación de microorganismos presentes en el polvo de madera por aserradero.

\begin{tabular}{|c|c|}
\hline Aserradero & Identificación bacteriana \\
\hline $\mathrm{Cl}$ & $\begin{array}{l}\text { Aspergillus sp. } \\
\text { Penicillium sp. }\end{array}$ \\
\hline $\mathrm{C} 2$ & $\begin{array}{l}\text { Aspergillus sp. } \\
\text { Penicillium sp. }\end{array}$ \\
\hline $\mathrm{ClO}$ & $\begin{array}{c}\text { Enterobacter sp. } \\
\text { Bacillus sp. }\end{array}$ \\
\hline $\mathrm{Cl}$ & Bacillus sp. \\
\hline $\mathrm{Cl} 2$ & Bacillus sp. \\
\hline $\mathrm{Cl} 3$ & Bacillus sp. \\
\hline $\mathrm{Cl} 4$ & Bacillus sp. \\
\hline
\end{tabular}


De la discusión anterior se desprende que hay un riesgo potencial de que los hongos y bacterias presentes en el polvo de madera afecten a los trabajadores de aserraderos y causen enfermedades, desde rinitis y alergias hasta infecciones más peligrosas, dependiendo de la susceptibilidad de cada individuo, sobre todo si se tiene en cuenta que la cantidad de polvo al que están expuestos es alta.

\section{Conclusiones y recomendaciones}

La empresa C5 presentó la concentración individual más alta del conjunto de datos para polvo de madera $\left(28,9 \mathrm{mg} / \mathrm{m}^{3}\right)$, superando el valor máximo permitido, que en el caso particular de esta empresa es de $0,70 \mathrm{mg} / \mathrm{m}^{3}$.

Los intervalos de confianza para el MLE de las empresas C2, C3, C5, C6, C8, CII, Cl2y Cl3 se encontraron por encima del TLV-TWA corregido por variaciones de jornada laboral en cada uno de los casos, indicando una situación de sobreexposición.

Los intervalos de confianza para el MLE de las tareas relacionadas con el uso de equipo eléctrico y el recibo de piezas procesadas se encontraron por encima del TLV-TWA sin corregir (igual a $1 \mathrm{mg} / \mathrm{m}^{3}$ ). No se pudo tomar una decisión sobre la situación de cumplimiento con la norma de la tarea de aserrío, por lo que no se descarta la posibilidad de que existan condiciones de sobreexposición.

Se encontraron especies de hongos y bacterias en las muestras de polvo de madera: Aspergillus, Penicillium y Bacillum. Todas ellas se reportan en estudios similares como comunes en aserraderos y como generadoras de trastornos en el tracto respiratorio (rinitis, alergias, alveolosis, etc.). No se descarta que la exposición a estos agentes biológicos contenidos en el polvo de madera puedan afectar la salud de los trabajadores, dado que las concentraciones de éste son altas en las empresas.

La caracterización más detallada de la presencia de agentes biológicos en el polvo de madera es un área de investigación novedosa en el país, que debe ser retomada en investigaciones futuras para una mejor comprensión del papel que desempeñan en las patologías respiratorias e inmunológicas del personal expuesto.

\section{Agradecimientos}

Los investigadores agradecen el apoyo financiero del Departamento de Gestión Empresarial en Salud Ocupacional del Instituto Nacional de Seguros y a la Vicerrectoría de Investigación y Extensión del TEC. También a las empresas dedicadas a la actividad de aserrío que aceptaron colaborar con el estudio y permitirnos realizar las evaluaciones.

\section{Bibliografía}

ACGIH. (20II). Threshold Limit Values and Biological Exposure Indices. Estados Unidos: Signature Publications.

Brief, R. \& Scala, R. (1975). Occupational Exposure limits for novel work schedules. Am. Ind. Hyg. Assoc. J., 76, 467-476.

Demers, P., Teschke, K., Davies, W., Kennedy, S. \& Leung, V. (2000). Exposure to dust, resin acids, and monoterpenes in softwood lumber mills. American Industrial Hygiene Association Journal, 6 I (4), $521-528$.

Douwes, J., Thorne, P., Pearce, N., Heederik, D. (2003) Bioaerosol Health Effects and Exposure Assessment: Progress and Prospects. Ann. Occup. Hyg., 47( 3), 187-200.

Fransman, W., McLean, D., Douwes, J., Demers, P., Leung, V., \& Pearce, N. (2003). Respiratory symptoms and occupational exposures in New Zealand plywood mill workers. Annals of Occupational Hygiene, 47(4), 287-295.

Gilbert, R. (1987). Statistical Methods for Environmetal Pollution Monitoring. New York: Van Nostrand Reinhold.

Halpin, D., Graneek B., Turner-Warwick, M., \& Newman A. (1994). Extrinsic allergic alveolitis in a sawmill worker: case report and review of the literature. Occup Environ Med, 5I, 160-4.

Hameed, A., Khoder, M., \& Farag, S. (2000) Organic dust and gaseous contaminants at wood working shops. J. Environ. Monit., 2, 73-76.

Hausen, B \& Rothernborg, H. (198I). Allergic contact dermatitis caused by olive wood jewelry. Arch Dermatol, I I $7(11)$, 732-734.

Health and Safety Executive. (2000). MDHS 14-3: General methods for sampling and gravimetric analysis of respirable and inhalable dust. Methods for the determination of hazardous substances. Health and Safety Laboratories.

Hornung, R. \& Reed, L. (1990). Estimation of average concentration in the presence of non detectable values. Appl. Occup. Env. Hyg, 5, 546-5I.

IARC. (1995). IARC monographs on the evaluation of carcinogenic risks to humans. Reino Unido: World Health Organization.

Kauppinen, T., Vincent, R., Liukkpnen, T., Grzebyk, M., Kauppinen, A. \& Welling, I. (2006). Occupational Exposure to Inhalable Wood Dust in the Member States of the European Union. Annals of Occupational Hygiene, 50(6), 549-561. 
Land, C., Sostari, B., Fuchs, R., Lundstrom, H. \& Hult, K. (1989). Intratracheal Exposure of Rats to Aspergillus fumigatus Spores Isolated from Sawmills in Sweden. Applied and Environmental Microbiology, 55( I I), 2856-2860.

Moro, D. y Okiki, P. (2003). Health risks associated with human exposure to wood dust in some nigerian saw-mills. ASSET Series B, 2(2), I 17- 130 .

Nigel, B., Dilworth, M. y Summers, N. (2007). Occupational Exposure to Wood Dust in the British Woodworking Industry in 1999/2000. Ann. Occup. Hyg, 5 I (3), 249-260.

Osman, E. y Pala, K. (2009). Occupational exposure to wood dust and health effects on the respiratory system in a minor industrial estate in Bursa, Turkey. International Journal of Occupational Medicine and Environmental Health, 22(1), 43-50. doi: | 0.2478/v 1000 I-009-0008-5

Oppliger, A., Rusca, S., Charrie're, N., Vu Duc, T. y Droz, P. (2005). Assessment of Bioaerosols and Inhalable Dust Exposure in
Swiss Sawmills. The Annals of Occupational Hygiene, 49(5), 385-391.

Rappaport, S., y Selvin, S. (1987). A method for evaluating the mean exposure from a log normal distribution. American Industrial Hygiene Association Journal, 48(4), 374-379.

Spee, T., Van de Rijdt-van Hoof, E., Van Hoof, W., Noy, D. y Kromhout, H. (2007). Exposure to Wood Dust Among Carpenters in the Construction Industry in The Netherlands. Annals of Occupational Hygiene, 5 I (3), 24I-248.

Teschke, K., Demers, P., Davies, H., Kennedy, S., Marion, S. y Leung, V. (1999). Determinants of Exposure to Inhalable Particulate, Wood Dust, Resin Acids, and Monoterpenes in a Lumber Mill Enviroment. Annals of Occupational Hygiene, 43(4), 247-255.

Woods, B. y Calnan, C. D. (1976). Toxic woods. Br J Dermatol, 94(| 3 Suppl), I-97. 